\title{
Réparer plus que répliquer
}

Les imprimantes 3D, des machines opérables

Repairing over Replicating. 3D Printers as Operable Machines

\section{Camille Bosqué}

\section{(2) OpenEdition}

Journals

Édition électronique

URL : https://journals.openedition.org/tc/7964

DOI : $10.4000 /$ tc. 7964

ISSN : 1952-420X

Éditeur

Éditions de l'EHESS

\section{Édition imprimée}

Date de publication : 31 octobre 2016

Pagination : 220-235

ISBN : 9782713225291

ISSN : 0248-6016

Référence électronique

Camille Bosqué, «Réparer plus que répliquer », Techniques \& Culture [En ligne], 65-66 | 2016, mis en

ligne le 31 octobre 2018, consulté le 29 septembre 2022. URL : http://journals.openedition.org/tc/7964 ; DOI : https://doi.org/10.4000/tc.7964 


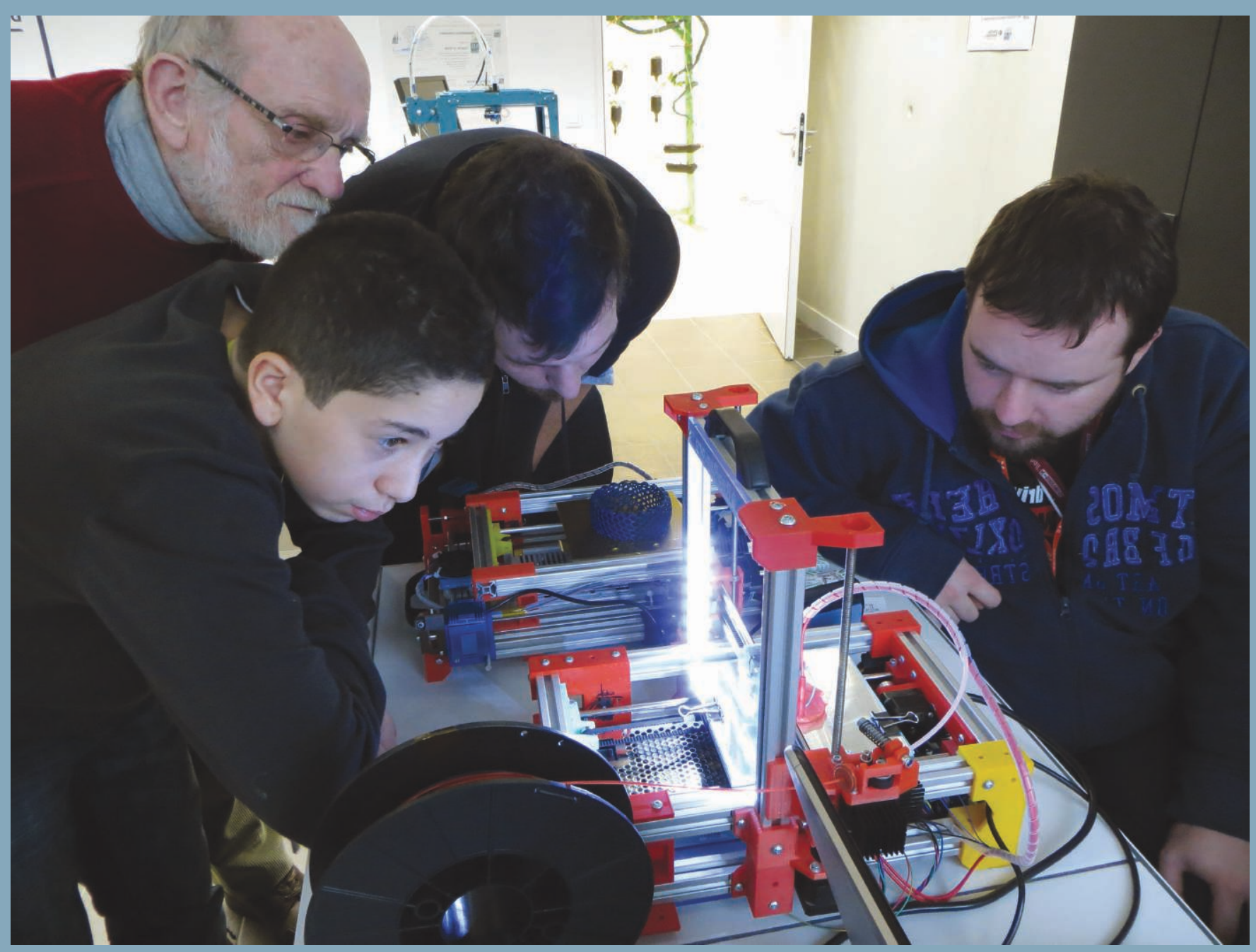




\section{Réparer plus que répliquer Les imprimantes 3D, des machines opérables}

Les technologies de fabrication additive existent dans la grande industrie depuis bientôt trente ans. Depuis une dizaine d'années, des modèles d'imprimantes 3D accessibles au grand public se développent et accompagnent l'essor du mouvement maker, qui défend une certaine conception de l'appropriation des technologies de fabrication et incarne pour de nombreux observateurs un terrain fertile pour une «nouvelle révolution industrielle» (Anderson 2012). Les valeurs de ce mouvement qui rassemble hobbyistes, designers, artistes ou amateurs croisent les questions de la fabrication à la demande, du partage des projets réalisés et de l'ouverture des technologies.

Il est désormais possible de construire soi-même ou d'acquérir une imprimante 3D d'entrée de gamme pour quelques centaines d'euros. De la même manière qu'en leurs temps l'intelligence artificielle ou la conquête de l'espace, l'impression 3D incarne le dernier progrès technoscientifique et à ce titre rassemble ou divise ses observateurs. De nombreuses publications n'hésitent pas à s'affoler devant la possibilité d'imprimer chez soi des pistolets en 3D, s'émerveillant ensuite devant la dernière prothèse de boîte crânienne fabriquée sur mesure pour un nourrisson (Mazzoli et al. 2009). Ces fantasmes se combinent dans les discours circulants avec la vision d'une «émancipation» du consommateur par une forme d'autofabrication. Dans le débat public, la qualité d'impression 3D disponible pour les milieux médicaux ou pour l'industrie aéronautique masque souvent la réalité assez pauvre de l'impression 3D par fil chaud, telle qu'elle se présente au sein des FabLabs (Gershenfeld 2005), hackerspaces, makerspaces et autres ateliers partagés. Les pratiques techniques émergentes comme l'impression 3D et plus largement la fabrication numérique personnelle incarnent les promesses d'une fabrication à petite échelle, sur mesure, donc sans déchet. Ces modes de production mettent en jeu des logiques économiques nouvelles. L'impression 3D fait partie du même arsenal de machines que la découpe laser ou le fraisage numérique. Le point commun de ces techniques tient à l'usage de programmes numériques qui permettent de concevoir des formes à l'aide d'un ordinateur. 
Cet article s'appuie sur une vaste enquête de terrain menée depuis 2012 au cœur des FabLabs, hackerspaces et makerspaces, dans le cadre d'un travail de thèse sur la fabrication numérique personnelle (Bosqué \& Ricard 2015). Une attention particulière a été accordée aux relations établies entre les habitants de ces espaces et leurs machines. Les logiques de développement de plusieurs projets et les techniques employées pour contourner certains obstacles ont également fait l'objet d'une attention particulière, puisqu'ils sont essentiels à ces pratiques.

L'objectif de cet article est de proposer une description critique des usages et des discours qui accompagnent le développement de l'impression 3D dite d'« entrée de gamme» et open source, en se concentrant sur certaines situations techniques qui présentent divers degrés d'émancipation et d'engagement. L'étude de cette technologie emblématique du mouvement maker et hacker révèle deux attitudes antagonistes dans les manières de faire avec ces machines: l'une tient à la production fascinée par réplication d'objets impensés et informés, et l'autre à une conduite plus réflexive d'ajustement et de réparation de ces machines elles-mêmes.

\section{Promesses et caprices d'une production à la demande}

La fabrication additive commence par la conception d'un modèle en 3D, qui est découpé en différentes couches par un logiciel. Les couches sont ensuite ajoutées par la machine une à une, chaque niveau s'associant au précédent. De nombreux objets peuvent désormais être «imprimés ». Sur la liste de ces nouveaux artéfacts, on trouve aussi bien des implants médicaux comme des couronnes dentaires que des jouets pour enfants, des pièces de voiture ou d'avion, des bijoux, des accessoires de mode, du mobilier, des coques de téléphones portables customisées, des bras bioniques et même des vaisseaux sanguins artificiels (Moskvitch 2011). Létendue des matériaux utilisables selon ce mode de conception et de fabrication augmente rapidement. Selon de nombreux spécialistes, « de futures innovations concerneront des machines capables d'imprimer ensemble des matériaux différents; d'imprimer des systèmes comme des batteries, des circuits ou des machines assemblées; d'imprimer organiquement des cellules-souches [...] et d'imprimer in situ dans le corps, dans l'espace, dans les océans profonds, ou en plein mouvement ${ }^{1}$. (Birtchnell \& Urry 2013).

Limpression 3D est une technologie encore en développement qui fait l'objet d'une spéculation collective sur de futurs scénarios. Ces idées ont donc inspiré de nombreux écrivains, dont Neal Stephenson dans son roman The Diamond Age (2012), Ian McDonald avec Brasyl (2007), Charles Stross avec Rule 34 (2012) et Cory Doctorow avec Makers (2009). Dans le roman de Neal Stephenson The Diamond Age (1995), on croise ainsi un «matter compiler», c'est-à-dire une machine capable de produire tous types de produits ou de nourriture à domicile, sans générer aucun déchet et en utilisant des nanotechnologies. Le futur de la fabrication numérique personnelle est souvent lié à des récits de science-fiction. Dans la première partie de Fabricated: The New World of 3D Printing (2013), Hod Lipson, qui est un chercheur à Cornell, publie un court texte intitulé «Everything Is Becoming Science Fiction », dans lequel il présente un futur proche où chacun pourra bientôt imprimer à domicile et à la 
demande toutes sortes de choses. Dans son récit, le personnage qui habite ce futur fantasmé imprime ainsi chaque soir sa propre brosse à dents customisée. Malgré ces promesses scintillantes d'un monde à venir dans lequel tous nos objets quotidiens pourraient être imprimés selon nos désirs les plus originaux, l'impression 3D n'a pas encore conquis tous les foyers. Néanmoins, dans la plupart des récits prospectifs qui entourent son développement, les possibilités offertes par l'impression 3D oscillent entre l'ambition d'une production à la demande mesurée, économique, locale, écologique et sans déchet, et une vision néoconsummériste qui encourage la production individuelle d'une multitude d'objets customisés, parfois appelés « crapjects» (une contraction de «crappy » et d'« objects»). Ceux-ci dévoilent le risque d'un monde envahi de «physical spams» (Townsend et al. 2011).

L'impression 3D fait partie d'un ensemble de technologies plus vaste. Selon Neil Gershenfeld, qui est ingénieur au MIT et dont les recherches sont à l'origine du mouvement des FabLabs aux ÉtatsUnis et dans le monde, la diffusion de cet accès aux technologies de fabrication numérique «permet à chacun de concevoir et produire des objets tangibles à la demande, n'importe où et à tout moment ${ }^{2}$.»

\section{Un monde de makers ordinaires}

Dans son livre FAB (2005), Neil Gershenfeld décrit un futur dans lequel chacun pourrait avoir accès à un «personal fabricator», une machine capable de produire n'importe quel objet mais surtout capable de fabriquer à son tour d'autres machines. Selon lui, «les articles scintillants sur les imprimantes 3D peuvent se lire comme les récits des années 1950 qui proclamaient que les fours à micro-ondes étaient le futur de la cuisine. Les micro-ondes sont pratiques, mais ils n'ont pas remplacé le reste de la cuisine (Solon 2013).»

En d'autres termes, l'impression 3D, selon Neil Gershenfeld, ne remplacera jamais les technologies de fabrication classiques - et soustractives - qui sont établies depuis longtemps. Néanmoins, la fabrication à la demande et la customisation d'objets pourraient encourager le développement d'économies nouvelles et devenir un argument compétitif pour de nombreuses sociétés. En effet, si n'importe qui était mis en capacité de produire ses propres objets, fort est à parier que nous assisterions à une prolifération décentralisée d'ateliers de fabrication et de petites cellules de microproduction, chacun répondant finalement à des demandes spécifiques. Ce que l'on appelle la « démocratisation de la production» pourrait alors avoir quelques points communs avec les récentes évolutions des marchés de la musique ou du film, pour lesquels le développement d'Internet a permis un accès plus large à une quantité de contenu et d'options de téléchargement (Rifkin 2001). Un tel scénario ou développement pour l'impression 3D pourrait alors se fonder sur l'échange et le partage de fichiers de modèles ou de blueprints (Lipson 2011). Ce sujet a été l'objet de nombreuses publications. En plus des répercussions profondes que ces technologies pourraient avoir sur l'industrie (Mills 2011, Karlgaard 2011), leur développement est souvent décrit comme capable de libérer une créativité populaire, en suivant le chemin tracé par la diffusion des ordinateurs personnels et d'Internet. C'est ce qu'indique Éric Von Hippel (2005): «L'usager qui innove peut développer 

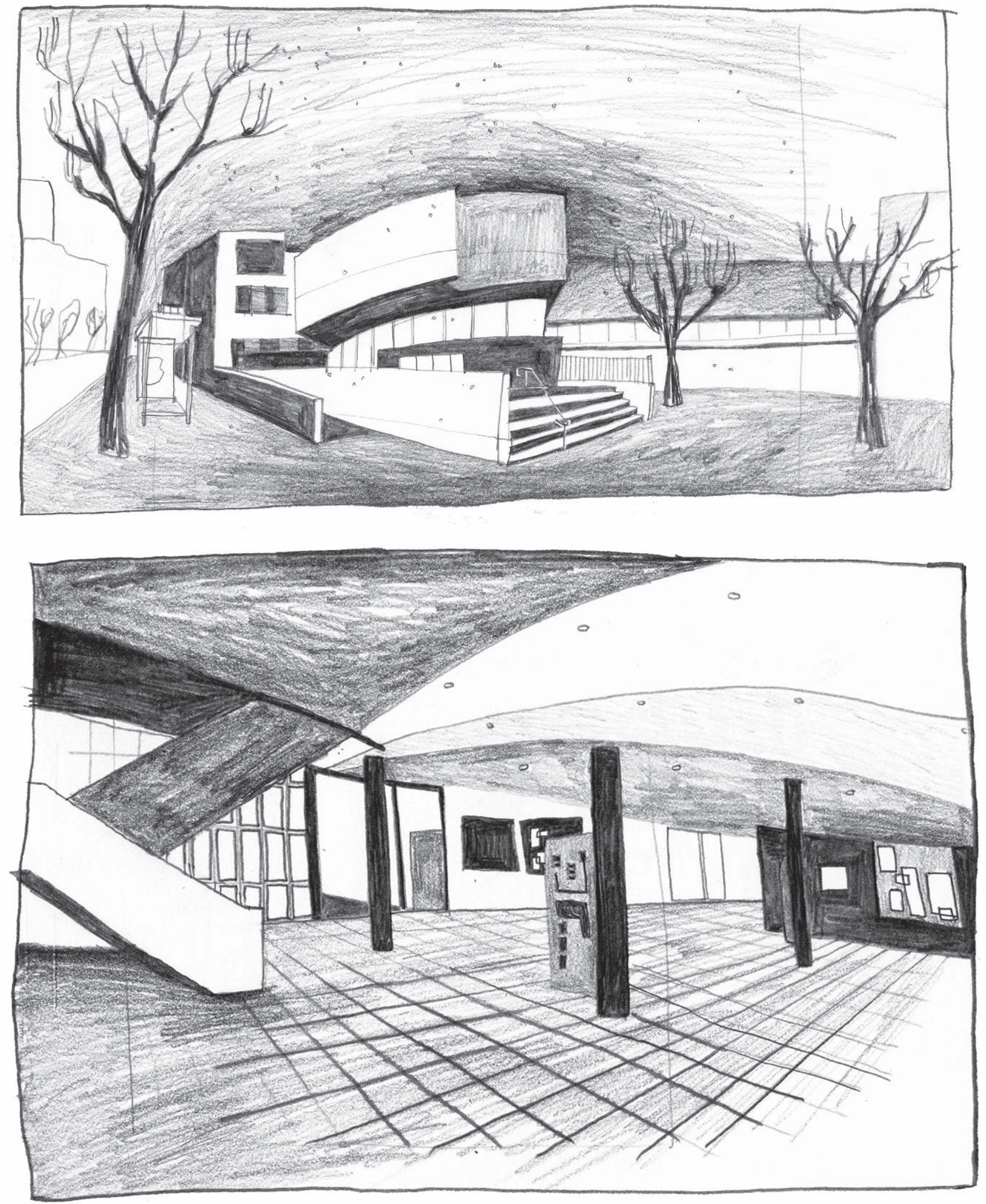


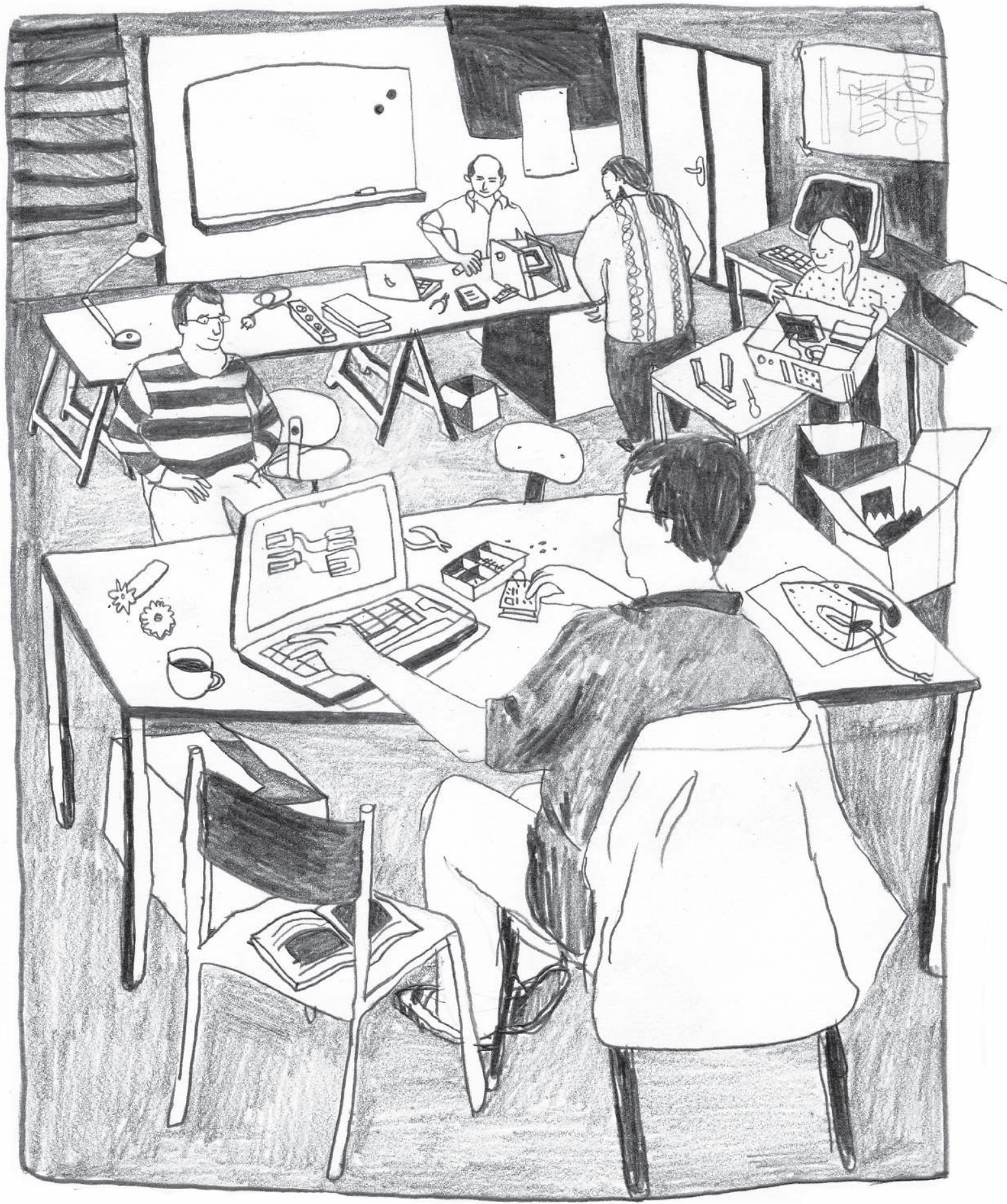



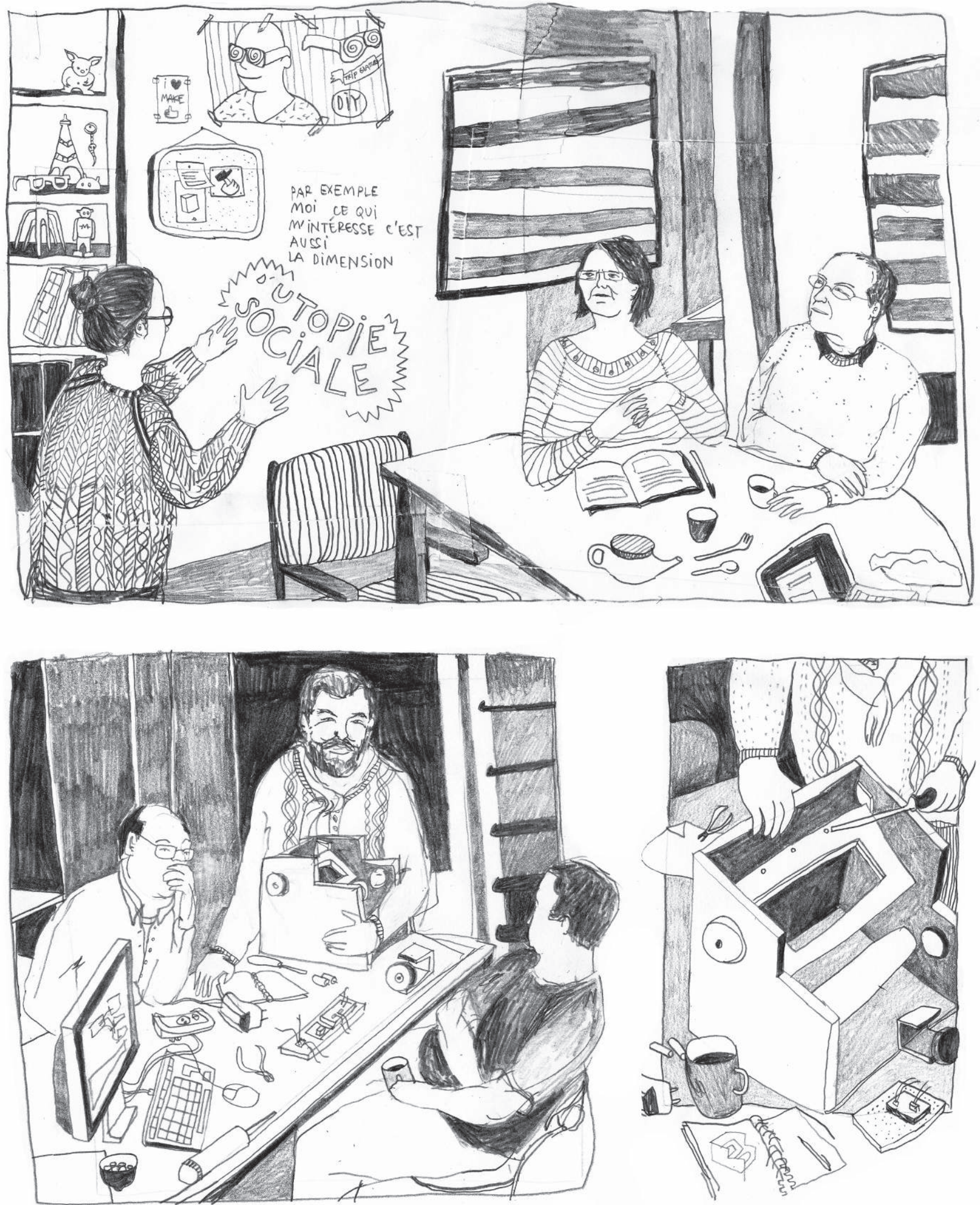


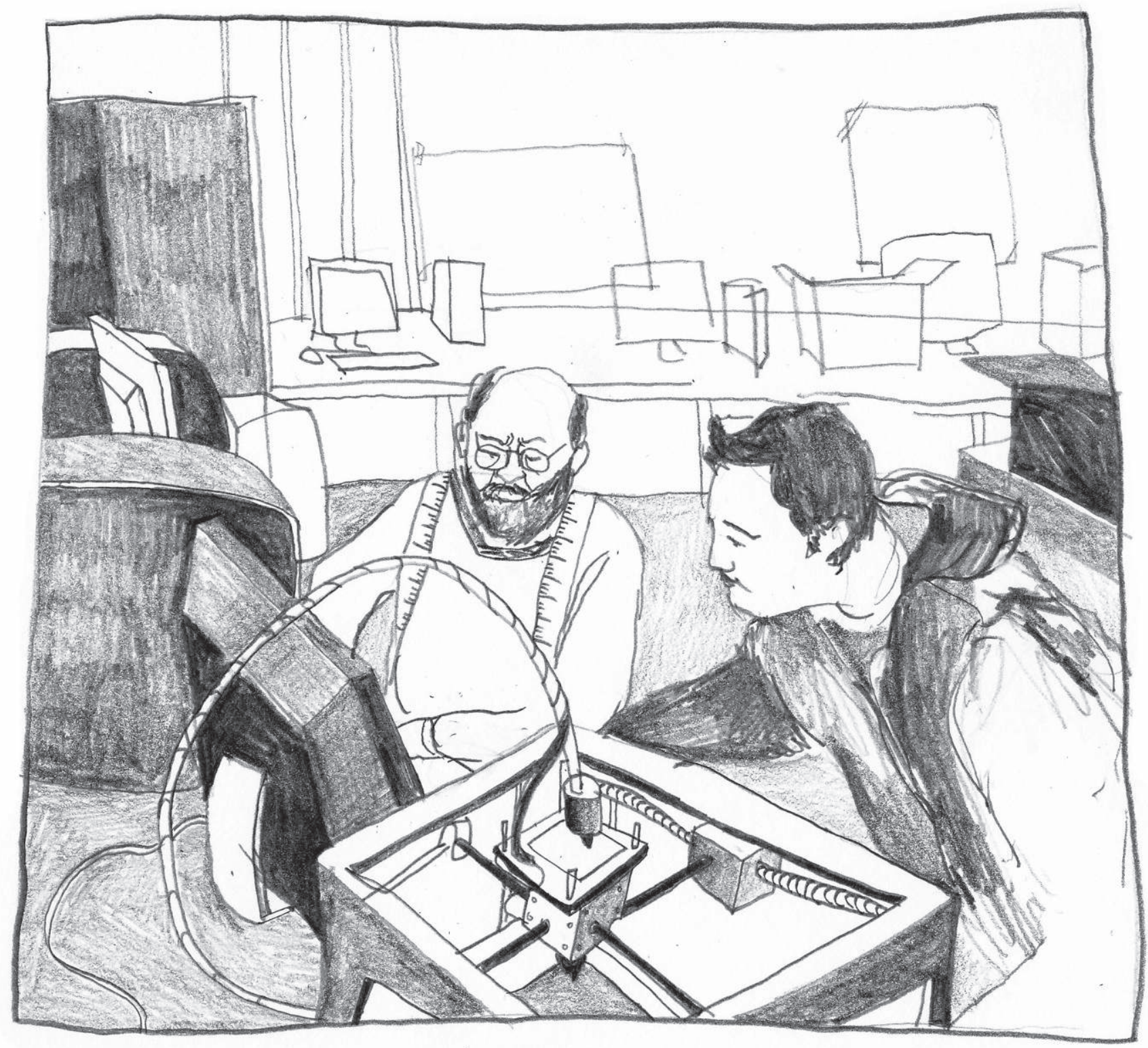


exactement ce qu'il souhaite, plutôt que de laisser les industriels agir comme ses agents (souvent assez imparfaits). De plus, les usagers ne doivent pas nécessairement tout développer eux-mêmes: ils peuvent bénéficier d'innovations développées et partagées librement par d'autres. »

Le développement de ces machines pourrait également mener à une décentralisation et à une distribution des moyens de production (Rifkin op. cit.).

L'accès à des techniques capables de traduire des fichiers électroniques en un objet tangible, combiné à la libre circulation de ces données, semble renouveler des pratiques de partage et de remix. Alors que des scientifiques ou industriels explorent à leurs niveaux les potentiels de la fabrication additive dans sa version la plus spécialisée, l'impression 3D se développe donc également au sein des FabLabs, hackerspaces et du mouvement maker, sous une forme open source. L'impression 3D, dans sa version d'entrée de gamme et telle qu'elle est employée par les makers et hackers est en effet l'un des outils principaux d'un mouvement plus large de bricoleurs, de militants et d'entrepreneurs.

L'exemple emblématique de l'imprimante 3D RepRap, entièrement conçue en open source, permet de mesurer la manière dont ces communautés s'emparent de la fabrication additive et envisagent de nouvelles manières de penser, qui oscillent souvent entre les premiers degrés de la réplication fascinée d'objets déjà modélisés et les ambitions plus pointues d'une conduite de réparation et d'ajustement.

\section{RepRap: un idéal communautaire d'autonomie et d'émancipation}

Des imprimantes 3D dites d'« entrée de gamme» et conçues en open source sont développées au cœur des FabLabs, hackerspaces et makerspaces par des communautés de hobbyistes que cette technique nouvelle rend curieux. La RepRap, qui est un exemple emblématique de ce type de machines, est ainsi loin d'être standardisée et présente certaines caractéristiques essentielles pour comprendre l'idéal d'autonomie et d'émancipation qui compose les fondements du mouvement maker. Johan Söderberg (2013) le formule de cette manière: «Faire fonctionner une machine correctement avec des résultats valables n'est pas une tâche triviale. [...] Cela requiert des compétences en soudure, mécanique, électronique, et aussi des notions de programmation.»

Adrian Bowyer, qui travaille au département d'Ingénierie à l'université de Bath, est l'inventeur de cette imprimante 3D open source. Son projet est né en 2005, sous le nom de RepRap, une abréviation de Replicating Rapid Prototyper. La réplication de cette machine est ainsi intrinsèquement liée à la manière dont elle a été conçue. Une RepRap est composée d'une structure métallique, assemblée avec des petites parties en plastique pensées pour être elles-mêmes formées par une autre imprimante 3D: impossible de s'en procurer dans une boutique de bricolage. En revanche, les moteurs, les parties électroniques et la buse d'extrusion ne peuvent pas être imprimés par une autre machine du même type. Le premier modèle d'imprimante 3D répliqué a été présenté en 2008, et décrit comme le «fils» de la toute première RepRap, assemblée grâce à des parties en plastique imprimées avec une RepRap identique.

Les FabLabs, hackerspaces et makerspaces sont des environnements fertiles pour le développement d'imprimantes 3D open source. Ce développement s'appuie sur un réseau virtuel 
et physique de développeurs et forme des communautés rhizomatiques au sein desquelles le partage et la discussion sur les expérimentations techniques autour du projet RepRap sont valorisés et reconnus. En dehors de toute hiérarchie ou subordination, les contributeurs du réseau RepRap collaborent d'une manière vertueuse, dans une logique d'organisation qui présente de nombreux points communs avec le «bazar» tel qu'il est décrit par Éric Raymond (1998) face à la « cathédrale» de l'organisation plus classique du travail collectif. Pour documenter les progrès de chaque version du projet, des images, vidéos et tutoriaux sont proposés en ligne (Gilloz 2013). Des réunions ou rencontres sont également organisées, lors desquelles la quantité impressionnante d'informations postées en ligne est discutée, commentée et évaluée. Ces communautés forment une part conséquente des activités liées aux FabLabs et hackerspaces.

Adrian Bowyer (2006) qualifie le développement du projet RepRap de «marxisme darwinien »: «Le projet RepRap va permettre la possession révolutionnaire, par le prolétariat, des moyens de production. Mais cela se fera sans cette révolution bordélique et dangereuse, et même sans ces trucs industriels bordéliques et dangereux ${ }^{3}$.»

Une forme d'évolutionnisme pourrait effectivement permettre aux RepRap d'être plus accessibles et de tracer un chemin pour le développement plus vaste des activités des hackers ou des makers. Cela pourrait alors être une manière de dépasser la fascination populaire qui entoure encore souvent ces machines.

\section{Au-delà de la fascination et de la réplication}

«La liberté de conception que permet le prototypage rapide est immense et ses procédés sont capables de créer des formes qui dépassent l'entendement ${ }^{4}$ », écrit Hopkinson (2006) : «Avant l'avènement de ces technologies, l'espèce humaine avait-elle jamais connu une situation dans laquelle visualiser et concevoir un produit est plus difficile que de le fabriquer ${ }^{5}$ ? » Nous ne sommes qu'au début de l'exploration des possibilités offertes par les technologies numériques de conception et de fabrication et notamment de l'impression 3D, ce qui explique la fascination suscitée par les démonstrations de ces techniques.

La scène se répète à chaque fois. L'imprimante est posée sur une table, reliée à l'ordinateur par un câble ombilical enroulé qui se confond avec d'autres fils électriques qui composent sa structure, généralement laissée nue. De loin on entend déjà une scansion mécanique, un son électronique haché, rythmé par la répétition invariable de la même séquence de petits bruits organiques. Au milieu des gargouillis et toussotements de l'appareil, le plateau chauffant est un mince feuillet métallique qui concentre toute l'attention. Sur cette fine estrade se transfère bientôt l'image qui s'affichait, quelques secondes auparavant, sur l'écran de l'ordinateur. Le plateau est parcouru en tous sens par une pièce en plastique de laquelle coule un fin filament fondu. Celui-ci est immédiatement assimilé par les couches antérieures. Déjà un autre niveau de l'objet apparaît, dans une éclosion lente, un dévoilement au ralenti de formes non immédiatement 
reconnaissables. Le mouvement est subtil. Le regard de tous est happé par les minuscules étages formés par la matière qui s'élève sur la petite scène, au cœur de la machine.

Les imprimantes 3D mettent en jeu une production par ajout de matière, indépendante de la main de l'homme. Néanmoins, dans la qualité qui est offerte aujourd'hui au grand public, la durée des impressions est encore longue. Elle suspend le temps. La démonstration requiert une attention complète, l'observation du procédé d'impression lui-même apportant généralement une satisfaction suffisante et la concrétisation de l'objet final étant presque de l'ordre du bonus. Mais il faut pourtant bien imprimer quelque chose. Fruits d'une machine dont on veut simplement montrer à l'audience fascinée qu'elle «marche», qu'elle tourne, qu'elle «fonctionne», les imprimantes 3D dans leurs contextes de démonstration et de performance font exister une production stérile, impressions à vide qui multiplient têtes de Yoda, vases aux formes psychédéliques plus ou moins étanches, malheureux bustes de femmes, bracelets élastiques et autres bibelots qui ne servent que de «prétexte» pour justifier la mise en route de ces machines. La plupart du temps téléchargés sur Internet, ces échantillons ou crapjects sont imprimés pour faire face à l'idée paralysante de pouvoir imprimer «n'importe quoi ». La plupart des démonstrations d'impression avec ces machines se raccrochent le plus souvent à des formules toutes faites, qui représentent une version préparée très à l'avance - presque lyophilisée! - des possibilités de la fabrication personnelle.

Les objets répliqués à la chaîne par les imprimantes 3D lors des sessions de démonstration, que, dans un autre article, j'ai proposé d'appeler des objets " phatiques ${ }^{6} »$, sont des preuves physiques des capacités de production de la machine. Dans son étude sur des ateliers de fabrication numérique personnelle menés dans des écoles, le chercheur américain Paulo Blikstein (2013) dénonce certaines dérives. Selon lui, «puisque les machines de fabrication numérique peuvent générer de beaux objets en engageant très peu d'efforts, les enseignants devraient éviter les projets qui en font la démonstration rapidement (quick demonstration projects) et pousser les étudiants vers des directions plus complexes. »

Ce qu'il appelle le Keychain syndrome (syndrome du porte-clés) incarne un mécanisme très fréquent dans les pratiques développées autour des machines de fabrication numérique et de l'imprimante 3D en particulier. Faire des porte-clés était ainsi l'objectif de la première session de découverte avec la découpeuse laser qu'il propose à une de ses classes. "À la quatrième session, j'ai compris que quelque chose clochait : l'atelier était devenu une usine de fabrication de porteclés, et les étudiants refusaient de s'engager dans d'autres projets», explique Paulo Blikstein.

En valorisant le produit plutôt que le processus, ces étudiants simplifient l'aspect technique pour se concentrer sur un objet «trivial» qu'ils arrivent à produire de manière autonome, puisque répétée et encadrée. Réduites à leurs capacités de réplication, les imprimantes 3D employées dans ce type de situations récurrentes ne mettent pas en jeu leurs caractéristiques de réparation, qui sont pourtant essentielles à la manière même dont ces machines elles-mêmes ont été conçues. 


\section{Réparer plus que répliquer}

La diffusion de modèles préparés et prêts à imprimer remet en question l'émancipation de l'amateur par les techniques numériques telle que Patrice Flichy (2011) la valorise, en tant qu'acquisition modeste de compétences poussée par la passion. Selon la définition qu'établit Jacques Rancière (2008), l'émancipation se dessine également dans un «brouillage de la frontière entre ceux qui agissent et ceux qui regardent, entre individus et membres d'un corps collectif».

Formes de privation de l'acquisition et de la transmission réelle d'un savoir-faire, la diffusion et la production répétée et répliquée des petits objets que l'on croise dans tous les ateliers de fabrication numérique prolongent cette situation de spectacle et encodent du même coup un savoir-faire figé, qui empêche de voir le vide pourtant propice à faire jaillir de nouvelles idées ou de nouveaux objectifs.

Quand Adrian Bowyer démarre en 2005 le projet RepRap, il conçoit ses premiers développements en encourageant son évolution par la réappropriation virale de ses plans. Il ne se doute pas alors que dix ans plus tard, ce modèle aura développé une généalogie de machines toutes différentes mais fondées sur des évolutions bien précises de telles ou telles parties. La RepRap (et toutes les générations d'imprimantes 3D conçues à partir de ses plans) est ainsi à la fois réplicable, modifiable, réglable et réparable. Elle est, en ce sens, un appareil «soignable» et soignant, réparable et réparant. L'idée de réparation, c'est-à-dire d'amélioration ou de «soin porté à» est centrale pour le philosophe Pierre-Damien Huyghe, qui, dans le texte Plaidoyer pour une technique hospitalisable (2015), explique que «si nous acceptions de présupposer que ce que nous entreprenons de réaliser peut tomber en panne (est pour ainsi dire d'avance autorisé à cela) et doit par conséquent être conçu de manière réparable», ces manières de se conduire avec la technique révéleraient notre existence en nous permettant de l'éprouver, d'en faire l'expérience. Ces appareils, capables de déterminer des conduites actives, doivent être réparables: «Il importe [...] que nous puissions envisager de réparer le monde», écrit Pierre-Damien Huyghe.

Il y a sans nul doute quelque chose dans cette idée qui relève du «bricolage» tel que Levi-Strauss l'analyse dans La pensée sauvage (1962), c'est-à-dire un réajustement de «résidus de construction et de destruction antérieures». En ce sens, les pratiques que nous étudions relèvent bien des «ruses» décrites par Michel de Certeau (1980) qui produisent sans nécessairement capitaliser. De la réparation à la réappropriation, diverses manipulations permettent donc de déjouer la passivité souvent induite par le spectacle fascinant des imprimantes 3D en action.

\section{Le hack: vers des objets " opérables"}

On remarque en réalité plusieurs phases dans la conduite d'une impression 3D. Après la fascination des premières minutes - qui persiste la plupart du temps une fois que l'impression a été «lancée »-suit une autre séquence, qui résiste souvent à la patience des novices mais qui s'impose de manière systématique aux utilisateurs plus aguerris. Contrairement aux imprimantes classiques en 2D, que l'on charge de papier et qui déroulent les feuilles une à une dans l'obscurité d'une salle 
de reprographie, celui qui engage une impression 3D ne laissera que très rarement son imprimante seule. Au FabLab du South End Technology Center de Boston, un petit panneau l'indique d'ailleurs dans une formule sans détour: "If you are using the 3D printer, you must be at the 3D printer. »

En effet, le constat est récurrent: «lancer» une impression ne consiste pas à l'envoyer bien loin: il s'agit au contraire de rester à côté de la machine pour plusieurs minutes, souvent même jusqu'à ce que l'objet ait entièrement atterri sur le socle chaud et que la forme soit complètement accouchée.

Au-delà du spectacle plaisant du fil chaud se superposant avec précision sur la couche précédente, l'individu déjà familiarisé avec l'appareil sait qu'une grande partie de son rôle pour la suite des opérations repose sur la gestion de l'aléa, facteur intrinsèque aux bredouillements de la machine. Dans ce cas précis, l'arrivée de l'aléa constitue aussi bien un élément de déroute qu'une source de satisfaction, son anticipation étant l'occasion de spéculations diverses à chaque impression. Il peut s'agir d'un défaut de réglage venant du logiciel, d'une poussière sur la plaque chauffante ou toute autre sorte d'événements imprévus. L'imprévu déjoue alors l'inactivité de l'opérateur face à la machine et peut être de tout ordre. Il requiert l'intervention et l'expertise de l'opérateur, chargé d'équilibrer et gérer les perturbations. En réalité, donc, tout n'est pas réglé d'avance. Malgré une vision souvent simpliste de ces technologies qui sont décrites selon les logiques du «Plug and Play», où «il n'y a plus qu'à appuyer sur un bouton et à attendre», ces machines requièrent une forme de traduction de la part de ceux qui les manipulent. Placé face à une telle «machine ouverte», l'opérateur devient cet « organisateur permanent, interprète vivant des machines les unes par rapport aux autres» décrit par Gilbert Simondon (1958).

Les signes sont variables: il peut s'agir par exemple d'une irrégularité dans le diamètre du fil ou d'un court-circuit provoquant un ralentissement du chariot d'impression. Ces marges d'erreur sont une autre partie intégrante de la situation technique offerte par les imprimantes 3D telles qu'elles se popularisent dans les tiers lieux de fabrication. En résultent des déformations du modèle, des coulures imprévues, des imperfections, décalages ou accidents allant parfois jusqu'à l'arrêt de la machine. Selon Pierre-Damien Huyghe (2015), « nous ne pouvons véritablement nous conduire qu'en raison de l'existence de marges de manœuvre qui font [...] que tout n'est pas réglé d'avance».

Selon lui, «si nous ne pouvons pas vivre sans techniques, nous ne pouvons véritablement nous conduire, au sein même de ces techniques, qu'en raison de modalités particulières de débrayage ».

Débrayer, si on entend ce verbe dans son sens le plus mécanique, revient à désolidariser, découpler, créer un écart dans le moteur, régler autrement pour ajuster. C'est précisément ce que doit faire celui qui veille sur son impression en guettant ou traquant la panne: enrouler le fil de manière plus lâche, remettre en question le diamètre de la buse, modifier progressivement les paramètres de déplacement du chariot. Dans l'exemple de la production de porte-clés décrit par Paulo Blikstein et rapporté plus haut, on peut penser qu'en évitant totalement de s'engager dans de nouveaux projets les étudiants pris par la frénésie de la fabrication en quantité en viennent alors à s'enchaîner aux logiques de production et de profit sans qu'aucun écart ne soit plus pratiqué. À l'inverse, une «conduite» suppose que le fonctionnement de la machine puisse être ouvert, modifiable, interprétable, réparable, « débrayable». Selon Pierre-Damien Huyghe, «il s'agit de se proposer 
les uns aux autres en lieu et place d'utilités ready-made, fussent-elles très opératoires, des objets à chirurgie opérable dans des unités de soin aussi petites, nombreuses et dispersées que possible».

«On a lu le mode d'emploi, mais on l'a jeté», m'expliquait un jeune hacker du /tmp/lab à Vitry lors d'une série d'entretiens que j'ai menés en décembre2012. Les pratiques du hack tirent parti des degrés de débrayage et de réparation offerts par nos technologies. Au/tmp/lab comme dans la plupart des hackerspaces, les machines se présentent souvent sans carter, sans grillage, sans protection. On y observe un refus de toute forme de surveillance des comportements qui est décrite comme obstacle à l'accident et aux mouvements libres. Les appareils présents dans les ateliers où travaillent les acteurs du mouvement hacker ou maker sont souvent laissés toutes entrailles ouvertes, l'opérateur étant ainsi en contact direct avec le corps de la machine, à l'affût du moindre bruit anormal, engagé personnellement dans l'activité technique. Les imprimantes 3D comme les RepRap et ses descendantes présentent alors un dépouillement, une mise à nu qui garantit et provoque le débrayage, l'évolution et le jeu, en accentuant la plasticité de l'appareil qui peut devenir lui-même objet d'expérimentation et de test. Allant à contre-courant des utilisateurs qui s'en tiennent aux commandes prévues pour les «actants» imaginés par les concepteurs (Akrich 1993) et luttant contre l'objet fermé et la boîte noire, les hackers tels qu'ils ont été étudiés par Sherry Turkle (1984) se placent délibérément dans des situations « au bord de la catastrophe».

Au-delà des ajustements et des jeux de débrayage qui déterminent une conduite active, certains opérateurs cherchent à explorer délibérément, en mettant en difficulté leurs imprimantes 3D, des situations d'échec technique qui les amènent à pousser plus loin les limites de leur ingéniosité.

L'étude des imprimantes 3D d'entrée de gamme, en tant que technologie emblématique du mouvement maker et hacker, met en lumière tantôt une production fascinée, par réplication d'objets impensés et informés, tantôt une conduite plus réflexive d'ajustement et de réparation de ces machines elles-mêmes.

Après l'examen de situations fermées dans lesquelles les imprimantes 3D sont mises en scène à distance d'une petite foule d'individus passifs, j'ai proposé dans ce texte de mettre en avant les propriétés des imprimantes 3D en tant que machines réparables.

Le curseur de l'émancipation dans les opérations pratiquées avec les imprimantes 3D est donc ainsi avancé depuis le degré le plus bas de la fascination jusqu'au point d'une technique qui s'autorise à être «braconnable» et buissonnière, démontable, ouverte et ajustable. Toutes ces pratiques ou situations prennent corps dans un collectif d'usagers ou d'individus devenus opérateurs, acteurs de configurations techniques variées.

\section{Notes}

1. «Some future innovations are likely to include machines able to print mixed materials at the same time; the printing of active systems such as batteries, circuits (...) assembled machines; organic printing of

stem cells (...); and in situ printing inside the body, in space, in deep oceans, or whilst in motion. » 
2. «Allows individuals to design and produce tangible objects on demand, wherever and whenever they need them»

3. «So the RepRap project will allow the revolutionary ownership, by the proletariat, of the means of production. But it will do so without all that messy and dangerous revolution stuff, and even without all that messy and dangerous industrial stuff. »
4. «The design freedoms afforded by Rapid Manufacturing are immense and the processes are capable of creating mind boggling geometries.»

5. «Prior to the advent of these technologies, has mankind ever been in the situation where visualizing and designing a product is actually harder than making it?»

6. Voir « What are you Printing? Ambivalent Emancipation by 3D Printing», article publié dans le Rapid Prototyping Journal en 2015.

\section{I'auteure}

Camille Bosqué est une ancienne élève du département design de l'École normale supérieure de Cachan, diplômée de l'École Boulle, agrégée d'arts appliqués et docteure en Esthétique et Design. Ses recherches portent sur la fabrication numérique personnelle dans les FabLabs, hackerspaces, makerspaces.

\section{Iconographie}

Image d'ouverture. Une imprimante 3D pliante, au FacLab de Gennevilliers. C Camille Bosqué.

Dessins. (C) Camille Bosqué.

\section{Références}

Akrich, M. 1993 «Les objets techniques et leurs utilisateurs. De la conception à l'action », in Conein B., Dodier N. \& L. Thévenot dir. Les Objets dans l'action. De la maison au laboratoire, «Raisons pratiques » 4 , Paris: Éditions de l'EHESS: 35-58.

Anderson, C. 2012 Makers: The New Industrial Revolution. New York, NY: Crown Business.

Ashlee, V. 2010 «3D Printing Spurs a Manufacturing Revolution", The New York Times: nytimes. com/2010/09/14/technology/14print.html?ref= ashleevance

Birtchnell, T. \& J. Urry 2013 «3D, SF and the Future», Futures: 25-34.

Blikstein, P. 2013 «Digital Fabrication and 'Making' in Education: The Democratization of Invention» in J. Walter-Herrmann \& C. Büching dir. Fab Lab: Of Machines, Makers and Inventors. Bielefeld: Transcript Verlag.

Bosqué, C. 2015a «Enquête au cœur des FabLabs, hackerspaces, makerspaces. Le dessin comme outil d'observation», TechniquesE Culture 64 «Essais de bricologie. Ethnologie de l'art et du design contemporains » : 168-185: tc.revues.org/7579.

- 2015b «What are you printing? Ambivalent emancipation by 3D printing», Rapid Prototyping Journal 21(5): 572-581, DOI: 10.1108/RPJ-09-2014-0128.
Bosqué, C. \& L. Ricard 2015 FabLabs, etc. Les nouveaux lieux de fabrication numérique. Paris: Eyrolles.

Bowyer, A. 2006 «Wealth without Money. RepRap Wiki »: reprap.org/wiki/Wealth_Without_Money.

Burns, M. \& J. Howison 2001 « Napster fabbing: Internet delivery of physical products », Rapid Prototyping Journal 7(4): 194-196.

Certeau, M. de 1980 L'Invention du quotidien, tome I, Arts de Faire. Paris: Union Générale.

Doctorow, C. 2009 Makers. Londres: HarperCollins.

Flichy, P. 2011 Le Sacre de l'amateur. Paris: Le Seuil.

Gershenfeld, N. 2005 Fab: The Coming Revolution on Your Desktop. From Personal Computers to Personal Fabrication. New York: Basic Books.

Gilloz, E. 2013 « RepRap Family Tree» : reprap.org/wiki/ RepRap_Family_Tree.

Hopkinson, N., Hague, R. \& P.M. Dickens dir. 2006 Rapid Manufacturing: an Industrial Revolution for the digital age. New York: Wiley.

Huyghe, P.-D. 2015 «Plaidoyer pour une technique hospitalisable», in Sociétés services utilités / À quoi tient le design. Grenoble: De l'incidence.

Mc Donald, I. 2009 [2007] Brasyl. Montreuil: Bragelonne. Levi-Strauss, C. 1962 La pensée sauvage. Paris: Plon. 
Lipson, H. 2011 This Will Change Everything : newscientist. com/issue/2823.

Mills, M. 2011 «Manufacturing, 3D Printing and What China Knows About the Emerging American Century», New York: Forbes. /www.forbes.com/sites/ markpmills/2011/07/05/manufacturing-3d-printingand-what-china-knows-about-the-emerging-american-century/\#79fe3e68468e.

Moskvitch, K. 2011 «Blood Vessels Made on 3D Printer»: bbc.co.uk/news/technology-14946808

Rancière, J. 2008 Le Spectateur émancipé. Paris: La Fabrique. Raymond, E. 1998 «La cathédrale et le bazar»: linuxfrance.org/article/these/cathedrale-bazar/cathedrale-bazar_monoblock.html

Rifkin, J. 2001 The Age of Access: How the Shift from Ownership to Access Is Transforming Modern Life. Londres: Penguin.

Simondon, G. 1958 Du mode d'existence des objets techniques. Paris: Aubier.
Söderberg, J. 2013 «Automating Amateurs in the 3D Printing Community: Connecting the Dots Between 'Deskilling' and 'User-friendliness' » in Work Organ. Labour Glob. 7: 124-139: hal-enpc.archives-ouvertes.fr/hal-00871041.

Solon, O. 2013 « Digital Fabrication is so Much More Than 3D Printing», Wired Magazine: wired.co.uk/news/ archive/2013-03/13/digital-fabrication.

Stephenson, N. 2012 The Diamond Age. Londres: Penguin Books. Stross, C. 2012 Rule 34. New York: Ace.

Townsend, A., Lyn., J., Fidler, D., Crawford, M. 2011 «The Future of Open Fabrication », Institute for the Future: iftf.org/uploads/media/SR-1390_FutureOfOpenFab. FINAL_sm.pdf.

Turkle, S. 1984 The Second Self: Computers and the Human Spirit. Boston: MIT Press.

Von Hippel, E. 2005 Democratizing Innovation. Cambridge: MIT Press.

\section{Nota bene}

Les illustrations de cet article sont extraites d'un travail en cours, réalisé par Camille Bosqué dans la suite de l'enquête qu'elle a menée au cœur des FabLabs, hackerspaces et makerspaces pour sa thèse, soutenue en janvier 2016. Dans la continuité de sa pratique de l'enquête par le dessin (Bosqué 2015), nous présentons ici une amorce de récit par la bande-dessinée. Le récit graphique présente, sans voix off, l'arrivée de la chercheuse sur son premier terrain, le FacLab de Gennevilliers. Ce FabLab est situé dans une annexe de l'université de Cergy-Pontoise à Gennevilliers, au fond d'un couloir. Il est fréquenté par des amateurs, bricoleurs, designers, étudiants. On y trouve toutes les machines dédiées à la fabrication numérique, et parmi elles plusieurs modèles d'imprimantes 3D autour desquelles les habitués de l'endroit se regroupent pour expérimenter ou détourner les possibilités offertes par cette technologie encore émergente. La narration amorcée ici est un travail en cours, un état intermédiaire de traduction par le dessin de la recherche théorique.

Camille Bosqué a également co-écrit avec Adrien Pavillard une web série documentaire en huit épisodes, sur le mouvement maker et les formes artistiques qui se développent en lien avec les outils de la fabrication numérique: «Fais-le toi-même», accessible en ligne: creative.arte.tv/ fr/faisletoimeme.

Ci-contre, les images dessinées par Camille Bosqué sont extraites de GIF animés qui accompagnent la lecture en ligne de cet article, disponible sous conditions d'accès, sur le site de TechniquesE Culture 65-66 « Réparer le monde» : tc.revues.org/.

\section{Pour citer cet article}

Bosqué, C. 2016 «Réparer plus que répliquer. Les imprimantes 3D, des machines opérables», Techniques ECulture 65-66 « Réparer le monde. Excès, reste et innovation», p. 220-235.

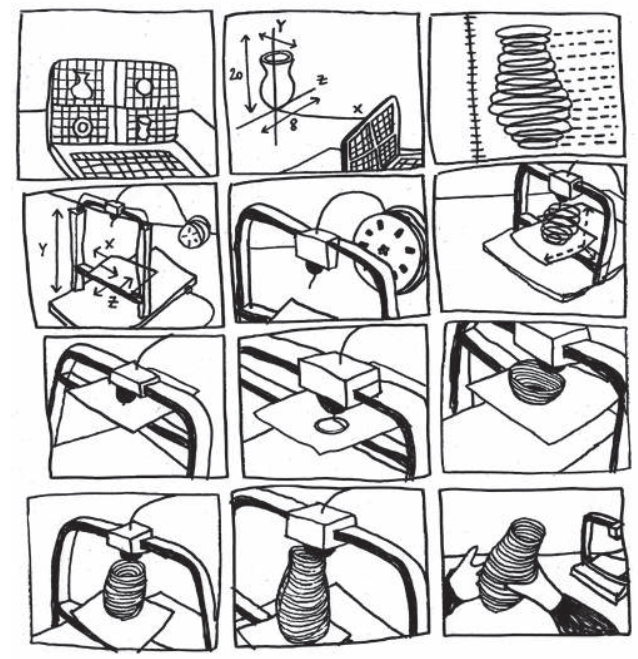

